\title{
Improving the precision of sea level data from satellite altimetry with high-frequency and regional Sea State Bias corrections.
}

\author{
Marcello Passaro $^{\mathrm{a}}$, Zulfikar Adlan Nadzir ${ }^{\mathrm{a}, \mathrm{b}}$, Graham D. Quartly \\ ${ }^{a}$ Deutsches Geodätisches Forschungsinstitut der Technischen Universität München, Arcisstraße 21, \\ 80333 Munich, Germany. Contacts: marcello.passaro@tum.de, +49 (89) 23031-1214 \\ ${ }^{b}$ Sumatera Institute of Technology (Itera); zulfikar.nadzir@gt.itera.ac.id \\ ${ }^{c}$ Plymouth Marine Laboratory; gqu@pml.ac.uk
}

\begin{abstract}
The sea state bias (SSB) is a large source of uncertainty in the estimation of sea level from satellite altimetry. It is still unclear to what extent it depends on errors in parameter estimations (numerical source) or to the wave physics (physical source).
\end{abstract}

By improving the application of this correction we compute $20-\mathrm{Hz}$ sea level anomalies that are about 30\% more precise (i.e. less noisy) than the current standards. The improvement is two-fold: first we prove that the SSB correction should be applied directly to the $20-\mathrm{Hz}$ data (12 to $19 \%$ noise decrease); secondly, we show that by recomputing a regional SSB model (based on the $20-\mathrm{Hz}$ estimations) even a simple parametric relation is sufficient to further improve the correction (further 15 to $19 \%$ noise decrease).

We test our methodology using range, wave height and wind speed estimated with two retrackers applied to Jason-1 waveform data: the MLE4 retracked-data available in the Sensor Geophysical Data Records of the mission and the ALES retracked-data available in the OpenADB repository (https://openadb.dgfi.tum.de/). The regional SSB models are computed parametrically by means of a crossover analysis in the Mediterranean Sea and North Sea.

Correcting the high-rate data for the SSB reduces the correlation between retracked parameters. Regional variations in the proposed models might be due to differences in wave climate and remaining sea-state dependent residual errors. The variations in the empirical model with respect to the retracker used recall the need for a specific SSB correction for any retracker.

This study, while providing a significantly more precise solution to exploit high-rate sea level data, calls for a re-thinking of the SSB correction in both its physical and numerical component, gives robustness to previous theories and provides an immediate 
improvement for the application of satellite altimetry in the regions of study.

Keywords: Satellite Altimetry, Sea State Bias, Sea Level, Retracking;

\section{Introduction}

Satellite altimetry measures the distance between the sea surface and the satellite (range), but this first estimate needs to be corrected for a number of geophysical effects, prior to being used for sea level estimation. The sea state bias (SSB) is among the time-variable corrections that are applied to sea surface height estimates from satellite altimetry. With a mean of $5 \mathrm{~cm}$ and a time-variable standard deviation of 2 to $5 \mathrm{~cm}$ in the open ocean (Andersen \& Scharroo, 2011), it is currently one of the largest sources of uncertainty linked with the altimetric signal (Pires et al., 2016).

Previous studies have usually identified different effects that play a role in the SSB. The first, the Electromagnetic (EM) bias, is strongly dependent on the significant wave height $(\mathrm{SWH})$ in the viewing area of the altimeter, and is due to the different backscattering of troughs and crests of the waves, which causes the EM range (what the altimeter actually measures) to be biased towards the troughs in comparison with the mean sea level (Fu \& Cazenave, 2001).

The second contribution is known as "Skewness Bias", which is related to the notion that the algorithms (retrackers) that are used to fit the altimetric waveform assume that the vertical distribution of specular reflectors illuminated by a radar altimeter is Gaussian, while their actual probability density function has a non-zero skewness.

The third contribution, historically called Tracker Bias, is actually a sum of errors related to the way the altimeter tracks the returning echoes. This contribution plays a role in the total SSB correction due to the empirical way in which this is estimated. Despite a few attempts to produce a theoretical description of the EM bias, e.g. Elfouhaily et al. (1999), any SSB correction currently used in the production of sea level data is derived by an empirical method that models this correction by expressing sea level residuals as a function of SWH and wind speed estimated by the altimeter itself. More recently, attempts have been made to add a third parameter, namely the mean wave period from a numerical model (Tran et al., 2010). The empirical nature of the SSB modeling implies that any sea-state dependent error in the residuals will be included in the correction.

Conceptually, only the third term varies with instrument and retracking algorithm, 
whilst the first two components should be the same for all $\mathrm{Ku}$-band altimeters. Two fundamental studies have dealt with this contribution. Firstly, Sandwell \& Smith (2005) has shown that part of the SSB correction is related to the inherent correlation between arrival time and rise time of the leading edge of the altimetric waveform, from which the physical parameters of SWH and sea level are estimated. Secondly, Zaron \& DeCarvalho (2016) developed a correction to de-correlate SWH and sea level estimations based on the analysis of their errors. They derived a correction to be applied to low frequency (LF, i.e. at $1 \mathrm{~Hz}$, corresponding to roughly one measurement every $7 \mathrm{~km}$ ) data that are already corrected for SSB. Quartly et al. (2016) demonstrated that the correlation of the errors in the estimation process shows up as correlated high frequency (HF, i.e. at $20 \mathrm{~Hz}$ for Jason-1, Jason-2 and Jason-3) SWH and SLA estimates within the LF spacing. A term related to issues in the fitting of a waveform cannot be considered as a SSB in a physical sense, since the non-linearities of the ocean waves should not vary at scales smaller than $10 \mathrm{~km}$. Nevertheless, due to the empirical derivation of the SSB models, it does influence any attempt in finding a parametric relation between SLA and SWH. For clarity and in analogy with Zaron \& DeCarvalho (2016), we will refer to "retracker-related noise" to discuss the contribution of this term to the total SSB correction.

In the empirical estimation of the SSB, the sea level residuals are analysed by differencing repeat measurements along collinear tracks (Chelton, 1994) or at orbit crossover points (Gaspar et al., 1994), or directly observing the anomalies with respect to the mean sea level (Vandemark et al., 2002). The residuals are modelled with respect to the variables influencing the sea state either in a parametric formulation (Fu \& Glazman, 1991; Pires et al., 2016) or non-parametrically solving a large linear system of observation equations for the SSB taken as unknown (Gaspar et al., 2002).

The motivation of this study is three-fold:

1. The SSB correction in the standard products, as any other geophysical correction, is given at LF, rather than at HF. Lately, the attention of the scientific community and particularly the effort to better observe coastal dynamics at a regional scale has moved to the exploitation of HF data (Cipollini et al., 2017b; Birol \& Delebecque, 2014). Gómez-Enri et al. (2016) and Passaro et al. (2018) have successfully applied the SSB model of the Envisat and ERS-2 satellite missions to high-rate estimations of SWH and wind speed from the ALES retracker (Passaro et al., 2014), although 
no SSB-specific consideration was made in analysing the results.

2. Several retrackers alternative to the standards have been proposed in recent years (Cipollini et al., 2017a). It is likely that different retrackers would bring different errors that play a role in the tracker bias. Nevertheless, for none of these alternative methods has a specific SSB correction been derived.

3. Several dedicated altimetry products during recent years provide region-specific processing (Birol et al., 2017; Passaro, 2017). Also the current phase of the European Space Agency's Sea Level Climate Change Initiative project (SL cci)(Quartly et al., 2017; Legeais et al., 2018) is focused on regional sea level analysis. Residual errors in the sea level, which are mirrored in the SSB model estimation, can also be dependent on the region. Since SSB models are estimated globally, regional predominance of certain wind and wave conditions might not be well enough represented in the realization of a global SSB model. An attempt of a regional SSB derivation was the SSB correction proposed for Cryosat-2 mission in the Indonesian Archipelago by Passaro et al. (2016), but comparison was not possible given that there is no official SSB model for that mission.

For these reasons, we aim in this work at computing a high-frequency, regional and retracker-dependent SSB correction in order to improve the performances of HF altimetry data. This is done in two subsequent steps. Firstly, we show that a simple application of the existing SSB model using HF estimations of two different retrackers is sufficient to reduce the SLA noise level in a comparable way to the correction of Zaron \& DeCarvalho (2016). Secondly, a new retracker-specific regional parametric SSB model is derived in two test regions.

The novelty compared with previous studies consists in i) an approach to reduce the retracker-related noise starting from HF data rather than the LF of Zaron \& DeCarvalho (2016), ii) the adoption of regionally focused corrections as suggested by Tran et al. (2010) and iii) the provision of a SSB correction for the ALES retracker, which is the algorithm chosen for the current phase of SL cci.

The test regions are defined together with the data sources in section 2 ; the methodology for SSB derivation and analysis is described in section 3; results are presented and discussed in section 4; the work and its perspectives are finally summarised in section 5 . 


\section{Data and Region of Study}

In this study HF observations from the Jason-1 mission are used. By choosing this mission, 7 years of data (January 2002 to January 2009) including cycles 1-259 (before the start of the drifting phase) can be exploited and at the same time comparisons can be made with the latest studies focused on SSB (Tran et al., 2010; Pires et al., 2016). The HF $(20 \mathrm{~Hz})$ data were extracted from the DGFI-TUMs Open Altimeter Database (OpenADB: https : //openadb.dgfi.tum.de) and are publicly available upon request. The OpenADB contains data from the original Sensor Geophysical Data Records (SGDR Version E) and from the Adaptive Leading Edge Subwaveform (ALES) reprocessing.

The SGDR product provides the orbital altitude, all the necessary corrections to compute the sea level anomaly and the output of the MLE4 retracker (Amarouche et al., 2004; Thibaut et al., 2010): range, SWH and backscatter coefficient. These are also estimated and given as output of ALES (Passaro et al., 2014). We computed the wind speed starting from the backscatter coefficient from the two retrackers using the processing described in Abdalla (2012).

The sea level anomalies (SLA) are derived from the range measurements using exactly the same orbital altitude and corrections (for tides and atmospheric effects), except, of course, the SSB correction, for both SGDR and ALES. Unrealistic estimations are identified using the outlier rejection suggested by Picot et al. (2003). Moreover, since the MLE4 retracker is not optimised for coastal waveforms, data within $20 \mathrm{~km}$ of the coast are excluded from the analysis.

The regions of study are the Mediterranean Sea (Med) and the North Sea (NS) and are shown in Figure 1. These regions have been selected in the context of the SL cci for the high interest in regional sea level dynamics and the relatively abundant in-situ measurements. Moreover, in the context of this study, these choices provide the opportunity to test the results in two areas characterised by different bathymetry, tidal regime and sea state conditions. 

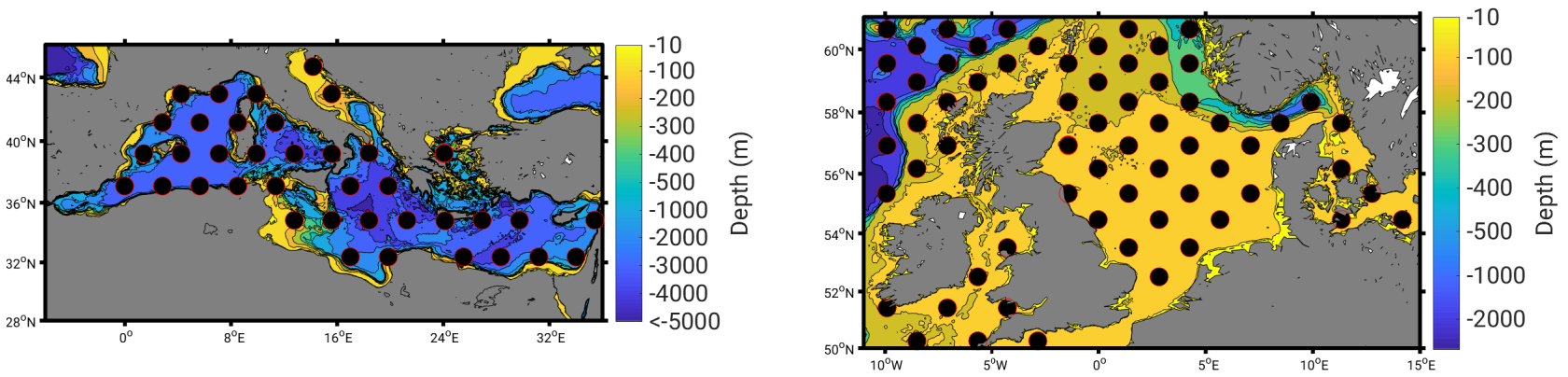

Figure 1: The two areas of study and their bathymetry. The black circles highlight the crossover locations used for the estimation of the regional SSB corrections.

\section{Methods}

\subsection{Different SSB corrections used in the study}

Three different SSB corrections are applied to derive the SLA in this study:

- $1-\mathrm{Hz}$ SSB is the SSB correction available at LF in the SGDR product. The correction is derived using the methodology described in Gaspar et al. (2002) and Labroue et al. (2004) and updated in Tran et al. (2010). This methodology adopts a non-parametric estimation: a statistical technique (kernel smoothing) is used to solve a large system of linear equations based on the observations and on a set of weights. The result is a $2 \mathrm{D}$ map of the SSB against wind speed and SWH.

- $20-\mathrm{Hz}$ SSB is the SSB correction derived by using the same 2D map from Tran et al. (2010) and obtained courtesy of Ngan Tran from Collecte Localisation Satellites, but computed for each HF point using the HF wind speed and SWH estimations from SGDR and ALES. As previously mentioned, the computation of the current SSB model is based on an empirical relationship between three retracked parameters. While part of it is due to the physics of the waves and will manifest itself at LF, the model contains also a relation that is due to the correlated errors in the estimation, which is performed at HF. This was already noted by Zaron \& DeCarvalho (2016), 
who stated that "the development of the SSB correction involves, in part, removing the correlation between SSH and SWH" and "it will have some impact on the shortwavelength components of these fields". Applying the SSB model at LF therefore means assuming that the error component of the sea level estimation related to the sea state exists only at long wavelengths, reducing its impact on the shortwavelength components. While recomputing a LF SSB model after eliminating the retracker-related noise must be an aim for future work, but goes beyond the scope of this paper, the original SSB model of the SGDR product is here applied at HF to consider its impact on the short wavelengths.

- Reg SSB is the SSB correction derived using the regional parametric models computed using the methodology described in 3.2 and then applied to each HF point using the HF wind speed and SWH estimations from SGDR and ALES.

\subsection{Derivation of regional SSB corrections}

Since the focus of this study is to investigate the improvements brought by the introduction of HF estimations and regional processing in the SSB derivation, we have not investigated the non-parametric modelling strategies, which are more complex to implement and numerically expensive. We chose instead a simple parametric form to model the regional corrections: the Fu-Glazman (FG) model proposed in Fu \& Glazman (1991), expressed as

$$
S S B=\hat{\alpha} S W H\left(g \frac{S W H}{U_{10}^{2}}\right)^{-\hat{d}}
$$

where $U_{10}$ is the wind speed computed from the backscatter coefficient estimated by each retracker, $g$ is the acceleration due to gravity, $\hat{\alpha}$ and $\hat{d}$ are the two parameters to be estimated.

This model incorporates a non-linear relation involving SWH and wind speed, so that finding $\hat{\alpha}$ and $\hat{d}$ at the same time is a non-linear problem. We linearise the problem by computing the $\hat{\alpha}$ coefficient for a set of $\hat{d}$ as in Gaspar et al. (1994).

Following the latter, the equations needed to compute the regional SSB models are built using HF SLAs at each crossover $m$ : 


$$
\Delta S L A_{m}=\hat{\alpha} X_{o}-\hat{\alpha} X_{e}+\epsilon
$$

where $o$ and $e$ stand for odd and even tracks (indicating ascending and descending tracks respectively), $\epsilon$ accounts for residual errors that do not depend on the missing SSB correction and:

$$
X_{o}=S W H_{o}\left(g \frac{S W H_{o}}{U_{10, o}^{2}}\right)^{-\hat{d}} \quad X_{e}=S W H_{e}\left(g \frac{S W H_{e}}{U_{10, e}^{2}}\right)^{-\hat{d}}
$$

We have therefore a set on $m$ linear equations, which we can express in vectorial form:

$$
\Delta \mathrm{SLA}=\hat{\alpha} \Delta \mathrm{X}+\epsilon
$$

Equation 5 is solved in a linear least square sense, giving one value of $\hat{\alpha}$ for each $\hat{d}$.

Finally, the chosen $\hat{\alpha}-\hat{d}$ couple is the one that maximises the variance explained at the crossovers, i.e. the difference between the variance of the crossover difference before and after correcting the SLA for the SSB using the computed FG model.

This derivation is shown in Figure 2 for SGDR and ALES in the two regions of study. The chosen $\hat{d}$ coefficients are indicated by a vertical line in the panels. $\hat{\alpha}$ is then derived as a function of $\mathbf{d}$. A discussion of these results is given in Section 4.2.

\subsection{Methods for data analysis}

\subsubsection{Methods for noise statistics}

Two noise statistics are employed to evaluate the precision of the dataset. Firstly, the high-rate noise is computed by considering the differences between consecutive HF SLA values, since SLA is not supposed to change significantly in 300 to $350 \mathrm{~m}$, which is the distance between one measurement and the next. This reference of noise was first used in Passaro et al. (2014) and subsequently employed in other studies, for example by Cipollini et al. (2017b).

Secondly, the difference in SLA variance between different datasets, i.e. SLA dataset corrected with the models in section 3.1, is computed on a 1-degree grid. Reducing SLA variance, both at global and regional scales, is the most common performance test 
a) SGDR

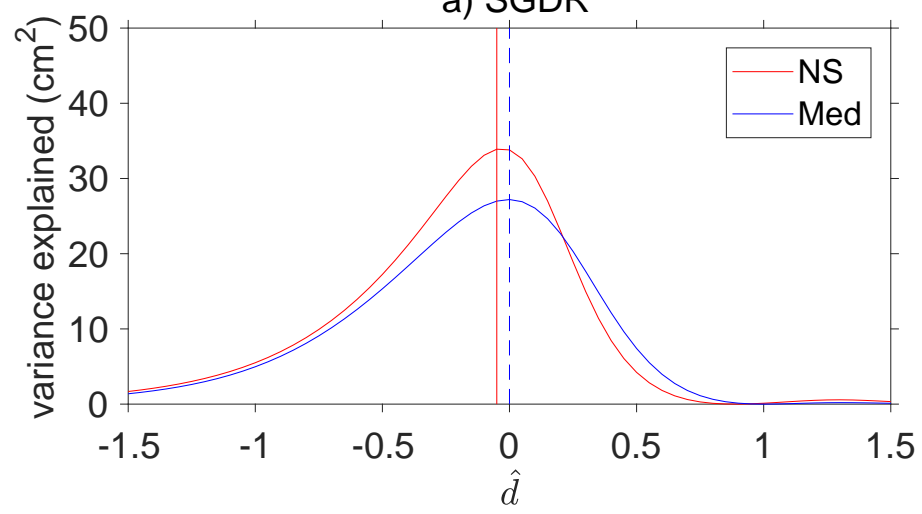

b) ALES

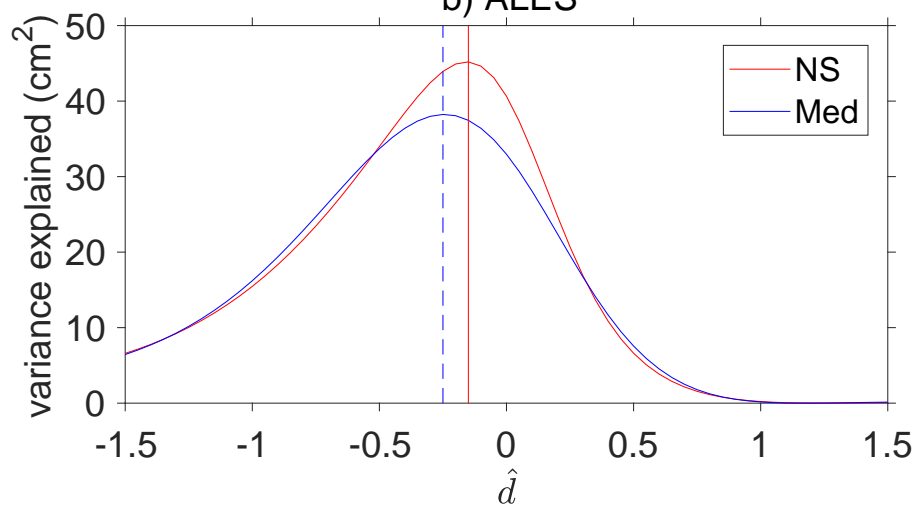

Figure 2: Parameter estimation for the FG model in the regions of study. Choice of parameter $\hat{\mathrm{d}}$ according to the variance explained by the application of the SSB correction at the crossover points for SGDR (a) and ALES (b) dataset. In all the plots, lines referring to the Med (NS) are specified in blue (red). Vertical lines highlight the optimal $\hat{d}$ value. 
for corrections applied to range measurements from satellite altimetry, for example wet tropospheric correction (Fernandes et al., 2015), inverse barometer correction (Carrère \& Lyard, 2003), dynamic atmosphere correction (Pascual et al., 2008). This metric has also been widely used in evaluation of SSB corrections (Tran et al., 2010); for our purposes we use the latest formulation proposed by Pires et al. (2016): the scaled SLA variance differences, which illustrate the impact of different SLAs relative to the regional variability, with the following formulation:

$$
S=\left[\frac{(\operatorname{var}(S L A 1)-\operatorname{var}(S L A 2))}{\operatorname{var}(S L A 1)}\right] \times 100
$$

\subsubsection{Intra-1Hz correlation}

Waveform data are subject to speckle noise leading to short-scale variations in the derived parameters. As this multiplicative noise is independent from one waveform to its successor, there is no correlation between the anomalies noted for consecutive records; however, any realization of the noise may affect multiple derived parameters in a concerted way. Variations in the trailing edge affect estimates of backscatter strength and mispointing in a highly correlated way (Quartly, 2009); variations on the leading edge have been shown to lead to synchronised errors in SWH and range (Sandwell \& Smith, 2005; Quartly et al., 2016).

The real values for SLA and for SWH will, in general, vary slowly over scales of $10 \mathrm{~km}$ (although there may be more pronounced changes close to the coast or rapidly shoaling bathymetry). Thus we consider 20 consecutive HF estimates of both parameters and calculate the regression coefficient within that ensemble, following the approach of Quartly et al. (2016). Most geophysical corrections (including the standard SSB model) are only applied at $1 \mathrm{~Hz}$, and so will not affect the connection between these terms. However, by choosing to apply the SSB model at $20 \mathrm{~Hz}$, we can evaluate how this affects the perceived connection between SWH and SLA.

\section{Results and Discussion}

\subsection{Robustness of the results}

When using a simple parametric model to estimate the SSB correction, its robustness will be influenced by the SWH and wind speed data distribution in the region of study. 
a )

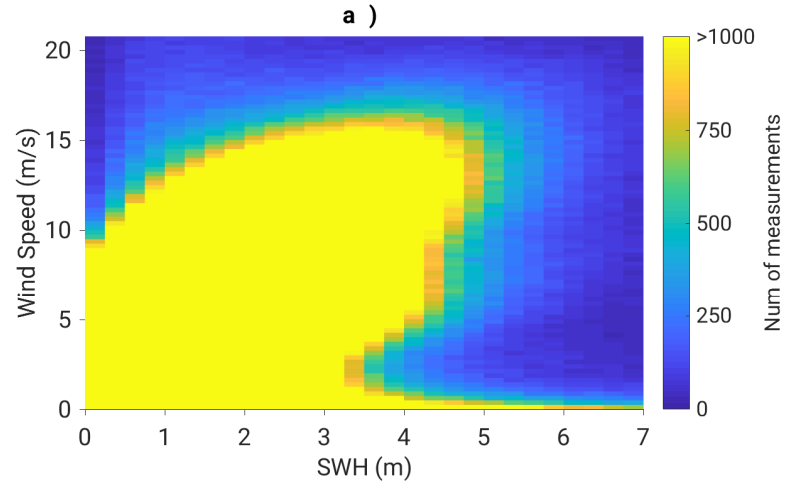

c)

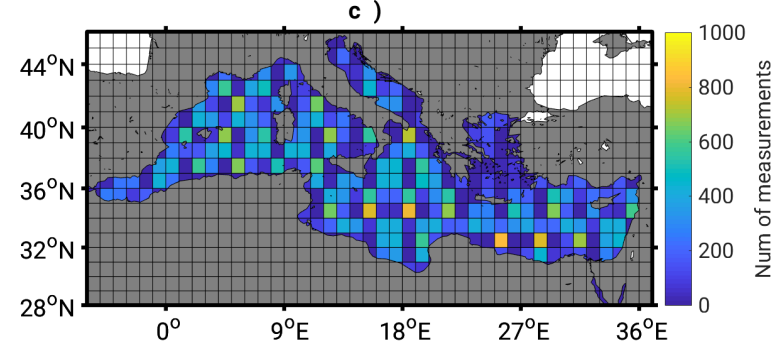

b )

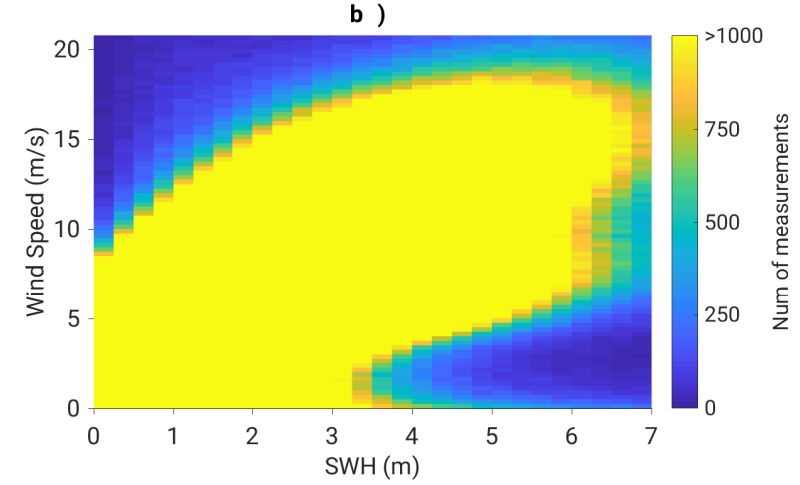

d )

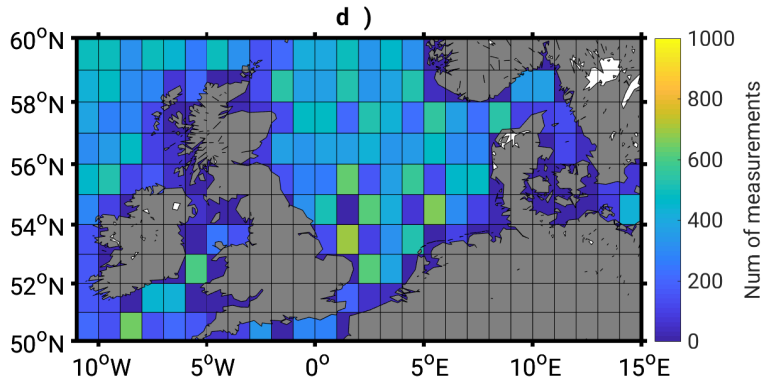

Figure 3: $\mathbf{a}$ and $\mathbf{b}$ ): 2d histogram of the number of measurements available for different wind and wave states in Med (a) and NS (a). The color bar is saturated at 1000 to show the limits of validity of the regional SSB corrections derived in this study. $\mathbf{c}$ and $\mathbf{d}$ show the locations of the valid measurements in a 1-degree grid.

Figure 3 gives us the possibility to understand the similarities and differences of the sea state characteristics in Med and NS. Panels $\mathbf{a}$ and $\mathbf{b}$ show the number of measurements for any wind-wave condition. There are in total over $10^{7}$ measurements in both regions, the color bar is saturated at $10^{3}$ measurements to highlight the conditions that happen rarely. Higher SWH conditions ( $>5 \mathrm{~m}$ ) are seen in NS more often than in Med, as expected, as well as stronger winds. The location of the measurements are reported on a 1-degree grid in $\mathbf{c}$ and $\mathbf{d}$, which is of course influenced by the Jason- 1 track pattern and by the fact that points closer than $20 \mathrm{~km}$ to the coast are not considered. This results in few observations in the Aegean Sea, because of the many islands within it.

\subsection{Comparison between models}

Figure 2 shows that the best parameterisation according to the FG model differs considerably between different retrackers (upper panel vs lower panel), while smaller differences are also seen between different regions. The stability and robustness of the 
solutions was confirmed by separately solving for maximum variance explained using just the first three years' data and also just the last four years' data, and noting that the results were essentially the same as the solution using all seven years' data. By using the best choice of coefficients, chosen as described in Section 3.2, the following Reg SSB models are defined:

$$
\begin{aligned}
& S S B_{S G D R, M e d}=-0.058 \times S W H\left(g \frac{S W H}{U_{10}^{2}}\right)^{0.00} \\
& S S B_{S G D R, N S}=-0.058 \times S W H\left(g \frac{S W H}{U_{10}^{2}}\right)^{0.05} \\
& S S B_{A L E S, M e d}=-0.050 \times S W H\left(g \frac{S W H}{U_{10}^{2}}\right)^{0.25} \\
& S S B_{A L E S, N S}=-0.061 \times S W H\left(g \frac{S W H}{U_{10}^{2}}\right)^{0.15}
\end{aligned}
$$

In order to better visualise the application of these models, Figure 4 displays the SSB correction to be applied according to each model to each HF SLA given a SWH and wind speed estimation. For comparison, the correction applied to the LF SLA in the official Jason-1 product is shown in panel a. To help the visualisation, SWH and wind speed intervals are restricted to the most frequent cases $(\mathrm{SWH}<5 \mathrm{~m}$, wind speed $<17 \mathrm{~m} / \mathrm{s}$ ). Panel $\mathbf{b}$ shows the spread between all the different models as standard deviation of the SSB values.

This figure and Equations 7 show that the set of optimal parameters is considerably different when switching retracker, at least for the parameter $\hat{d}$, which is responsible in the SSB for the influence of the wind speed estimation. The latter is considerably more influential on ALES than on SGDR. The dependence of the crossover differences on the sea state is therefore strongly influenced by correlated errors between the retracked parameters, as postulated in Sandwell \& Smith (2005). If the physics of the interaction between the signal and the waves were dominant with respect to the retracker-related noise, then the difference of coefficients and SSB model between ALES and SGDR would not be so marked. Regional differences are also present, although less prominent. On one side, these can be the consequence of the choice to model the SSB in a parametric form, which could influence the solution of the linear system due to the presence of more observations with higher sea states in NS. On the other side, other remaining sea-state dependent residual errors can play a role. In general, regional differences of the wave 
Table 1: Variance at crossover locations (XO var) before and after the application of the regional sea state bias (Reg SSB) correction based on the derived Fu-Glazman model. The last row provides the corresponding numbers reported in Gaspar et al. (1994) for a global solution using $1 \mathrm{~Hz}$ data.

Dataset SGDR Med SGDR NS

ALES Med

\section{ALES NS}

Gaspar et al. (1994)
XO var before SSB $\left[\mathrm{cm}^{2}\right]$

135.6

233.7

167.8

246.9

127.7
XO var after SSB $\left[\mathrm{cm}^{2}\right]$

108.4

199.8

129.8

201.8

120.4

climate from the global average exist and can justify differences between regional and global SSB models. For example, the prevailing difference between the regional SGDR SSB models of this study and the global model is a higher sensitivity of the former to the SWH, which means that for the same value of SWH the regional SSB will be in absolute value higher than in the global model. A comparable effect was found by Tran et al. (2010) in the same regions considering the mean difference between a 3-D SSB model including a dependence on the wave period and the global SSB model.

In Table 1 the variance at the crossover before and after the application of the SSB corrections is reported, together with the values reported by Gaspar et al. (1994), who estimated the coefficients of FG model on a global scale. The variance in the latter is smaller, since in our study we consider shelf seas and areas that are much more variable than the deep open ocean and since we use HF values at the crossover points, instead of LF as in Gaspar et al. (1994). The higher variance in ALES compared with SGDR corresponds to the known $1 \mathrm{~cm}$ difference in RMS for precision of HF estimations, as reported in Passaro et al. (2014). The models computed in this study decrease the variance at the crossover by 15 to $23 \%$. In comparison, the variance after the global LF correction by Gaspar et al. (1994) decreased by 6\%. This comparison is only meant to underline the different way in which the same parameterisation is estimated in this study with respect to previous literature. Considerations about precision are instead given in the next sections.

\subsection{Noise statistics}

In this section we study the performances of the SLA corrected by different SSB models using the statistics described in Section 3.3.1. 


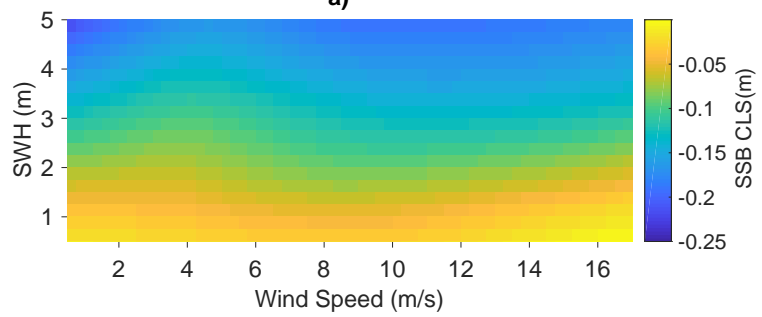

c)

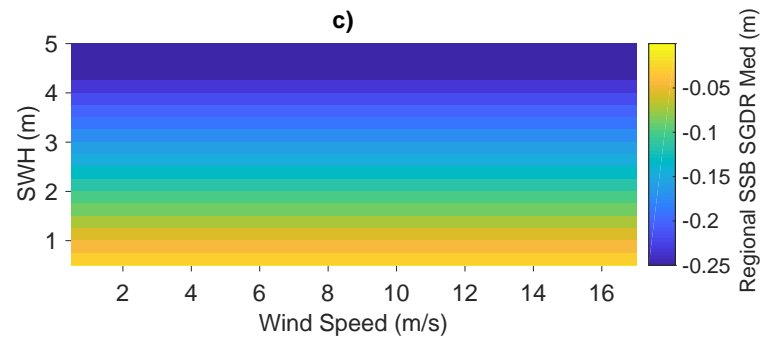

e)

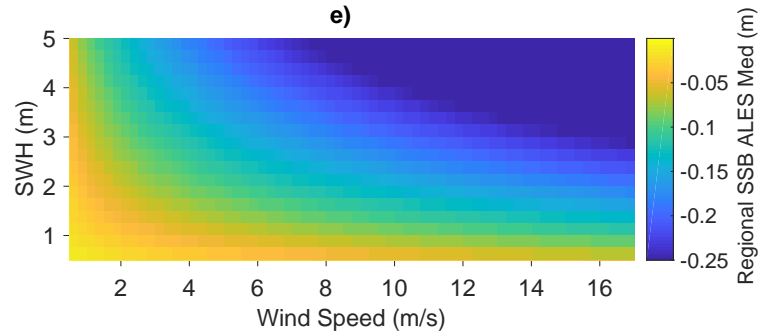

b)

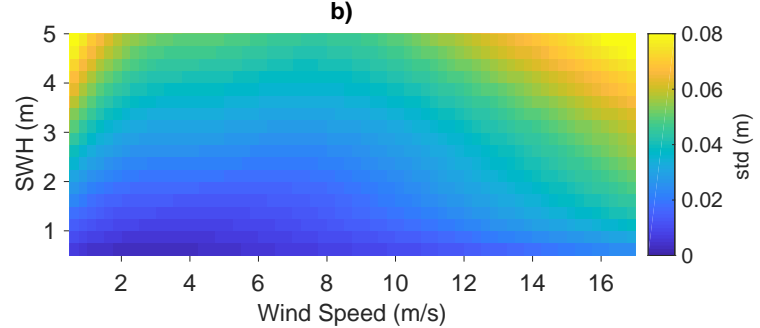

d)

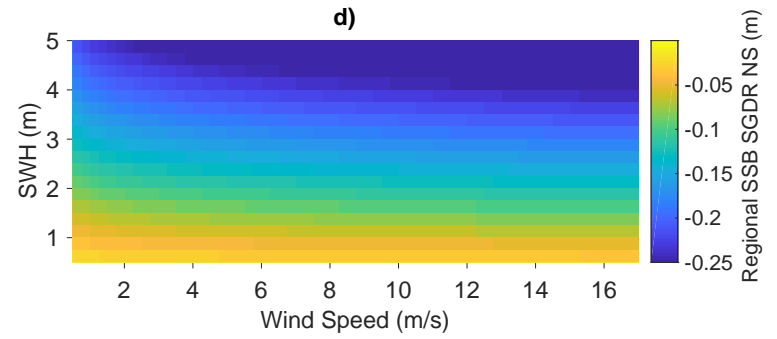

f)

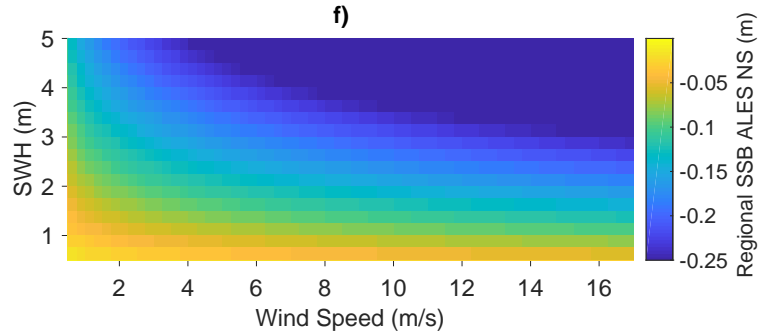

Figure 4: Different SSB models outputs used in this study for SWH-wind speed domain considering the same dataset and spread between them. (a) SSB model currently in use for Jason-1 SGDR. (b) Spread of the models in these figures, computed as standard deviation. Regional HF FG model for SGDR data in Med (c) and NS (d). Regional HF FG model for ALES data in Med (e) and NS (f) 
Firstly we consider the noise quantified as difference of consecutive HF SLA measurements. We estimate for each cycle the average noise binned in 25-cm intervals of SWH. Then, results are averaged over all the cycles and displayed in Figure 5 with respect to the SWH. The more irregular lines seen at higher SWH are due to the decrease in available measurements, as reported in the lower panels. The blue curves show the HF SLA noise in Med (a) and NS (b) when correcting ALES (dashed line) and SGDR (continuous line) with the given 1-Hz SSB. For the 1-cm difference between the two retrackers, we refer the readers to the considerations in the previous section. The behaviour of the curves in the Med is much more complicated than in the NS, whose shape is similar to the globally-averaged behaviour, which is shown for example in Garcia et al. (2014). This calls for a dedicated regional approach, in particular when estimating empirical corrections such as the SSB correction, but ultimately leading to a better understanding and parameterization of a global process.

The application of the $20-\mathrm{Hz}$ SSB decreases both the noise at low sea states and the slope of the noise curve. This corresponds to the effect observed by Garcia et al. (2014) when applying a 2-pass retracker to decouple SWH and range estimation and is again proof that SSB should be applied at HF, because it includes retracking errors that are strongly sea-state dependent. On top of that, further improvement of the same kind is brought when the Reg SSB models from Equations 7 are applied. Notably, the improvement is of a similar magnitude for both SGDR and ALES and therefore it is not only attributable to the need of a specific correction for a different retracker. This means that our regional high-frequency empirical parametrical SSB correction is superior to the global non-parametric SSB model, even if the latter is applied at HF. It must be stressed that the metrics used in this paper, which follow what is done in previous works on the corrections to the range estimated by radar altimetry, are focused on improvements of the precision, i.e. the repeatability of a HF sea level estimate, which can be quantified by a reduction in the HF variance. An evaluation of the improvement in accuracy shall rely on external data, such as tide gauges, and can be the subject of a future validation study involving other regions as well.

To better quantify this improvement, we compute the scaled SLA variance difference in the two regions of study on a 1-degree grid for SGDR in Figure 6 and for ALES in Figure 7. The median results are summarised in Table 2. The comparison is performed 

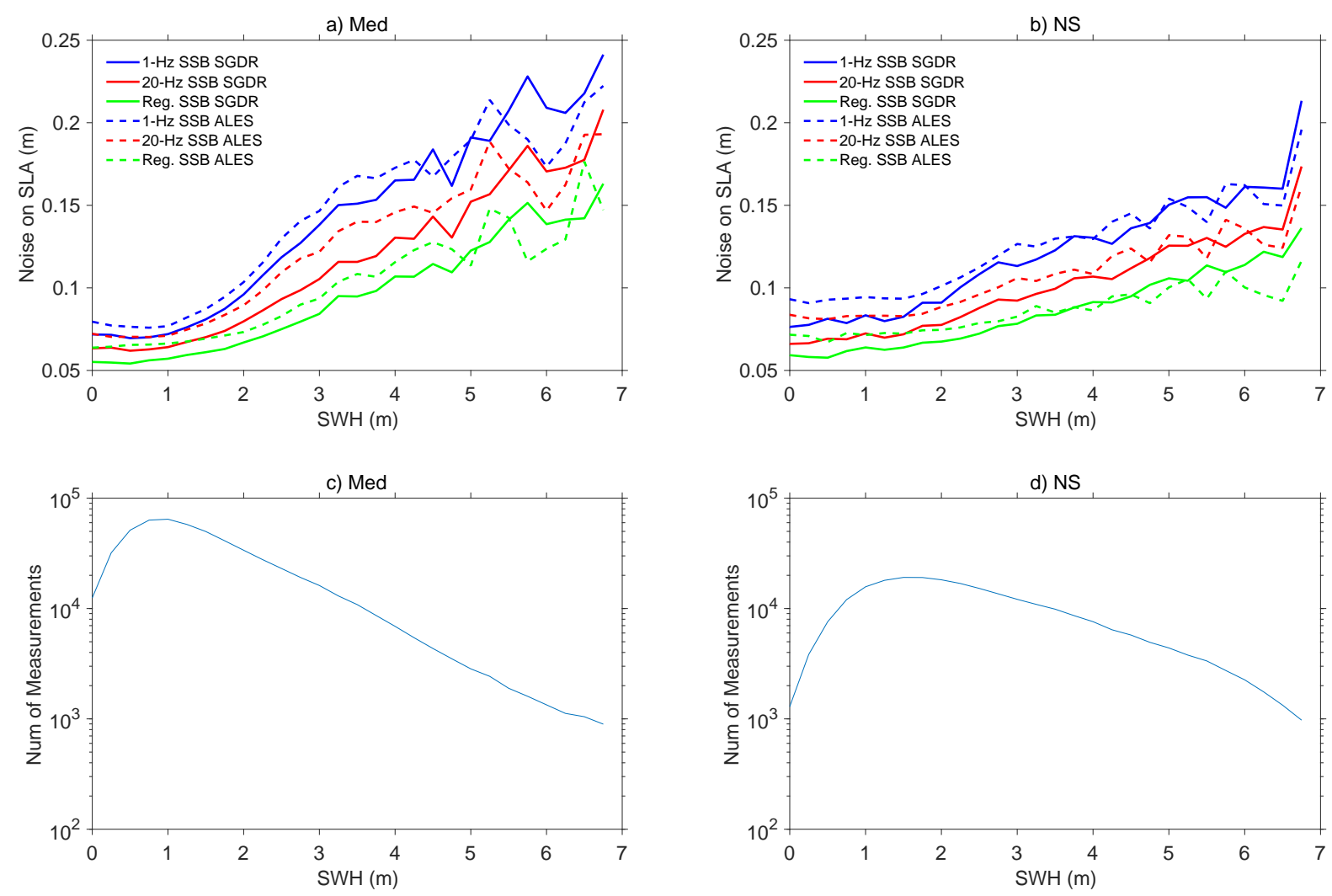

Figure 5: Noise of the sea level anomalies computed as difference between consecutive high-rate estimations using different SSB corrections analyzed in this study in Med (a) and NS (b). Continuous lines refer to SGDR data, while dashed lines refer to ALES data. The sea level anomalies were corrected with the original 1-Hz SSB correction (blue), with the 20-Hz SSB correction (red) and with the regional SSB correction (green). Number of measurements available with respect to the significant wave height in Med (c) and NS (d). 
Table 2: Median scaled SLA variance improvement in the regions of study. For each coloumn, the reference is the correction of the right and the challenger is the correction on the left. The percentage shows the improvement when using the challenger with respect to the reference.

Dataset 20-Hz vs 1-Hz SSB [\%] Reg vs 20-Hz SSB [\%] Reg vs 1-Hz SSB [\%]

SGDR Med

19.18

17.31

14.05

12.21
19.83

15.01

18.77

16.67
34.64

29.93

29.34

25.81

by choosing a reference and a challenger dataset: in this way, panels $\mathbf{a}$ and $\mathbf{b}$ show the performances of the $20-\mathrm{Hz}$ SSB taking the $1-\mathrm{Hz}$ SSB as a reference; panels $\mathbf{c}$ and $\mathbf{d}$ show the performances of the Reg SSB taking the $20-\mathrm{Hz}$ SSB as a reference; finally panels e and $\mathbf{f}$ shows the performances of the Reg SSB taking the 1-Hz SSB as a reference and therefore summarise the overall improvement given by this study against the current product. The improvements are of the same amount independently of the region and the variability, as already seen in the crossover statistics of Table 1, with the important addition that the decrease in variance is ubiquitous also within the domains. A few points present exceptions: they either correspond to locations in which very few observations are available (see Figure 3) and therefore might present residual outliers with high sea states (and consequently high SSB correction) or, interestingly, to locations characterised by a deep bathymetry in the NS (Figure 7, panels $\mathbf{d}$ and $\mathbf{e}$ ). The latter point is yet another hint as to the different characteristics of sea-state dependent altimetry errors for shallow areas and the necessity of a dedicated regional processing.

To summarise using the statistics in Table 2, results are very robust. The simple application of an SSB correction based on HF data improves the precision of HF sea level data by 12 to $19 \%$. We notice how the improvement shown by the $20-\mathrm{Hz}$ SSB for SGDR is similar to the one reported by Zaron \& DeCarvalho (2016) in their North Pacific test region, which indicates that this application is an alternative method to reduce the retracker-related noise. Subsequently, the recomputation of a parametric regional SSB model improves it overall by $26 \%$ to $35 \%$.

\subsection{Intra-1Hz correlations}

The regression coefficient $\beta$ between the $20-\mathrm{Hz}$ values for SLA and for SWH from the SGDR has a median value of -0.092 , with an inter-quartile range of -0.100 to -0.064 , 

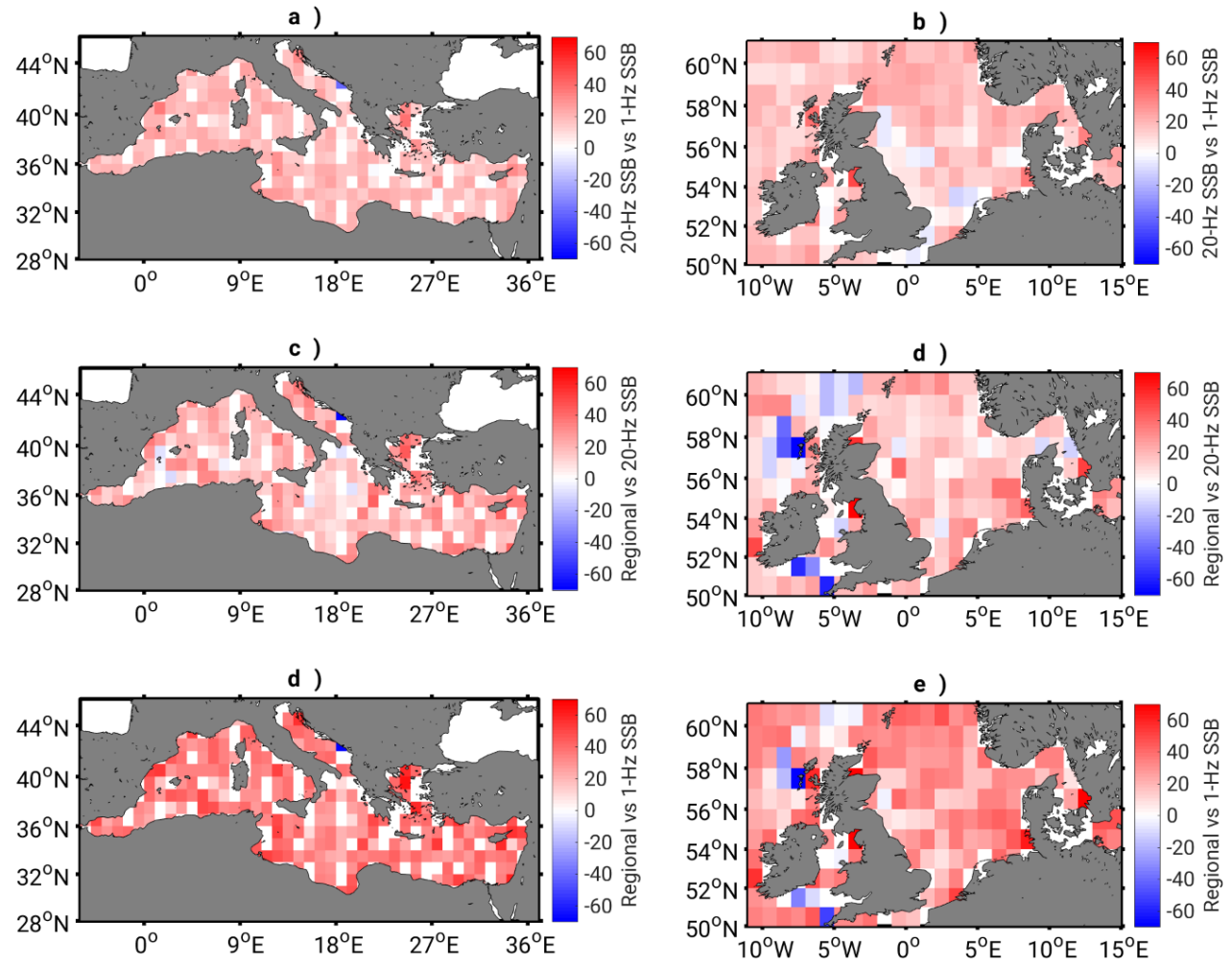

Figure 6: Percentage of scaled sea level anomalies (SLA) variance differences between a challenger and a reference model. a and $\mathbf{b}$ : SLAs computed with 20-Hz SSB correction (challenger) against the ones computed with the original $1-\mathrm{Hz}$ correction (reference). c and $\mathbf{d}$ : SLAs computed with $20-\mathrm{Hz}$ SSB correction (challenger) against the ones computed with the regional SSB correction (reference). $\mathbf{d}$ and $\mathbf{e}$ : SLAs computed with regional SSB correction (challenger) against the ones computed with the original 1-Hz correction (reference). Red squares represent regions with a lower SLA variance for the challenger, i.e. an improvement in the noise statistics with respect to the reference. The dataset used is the SGDR. 

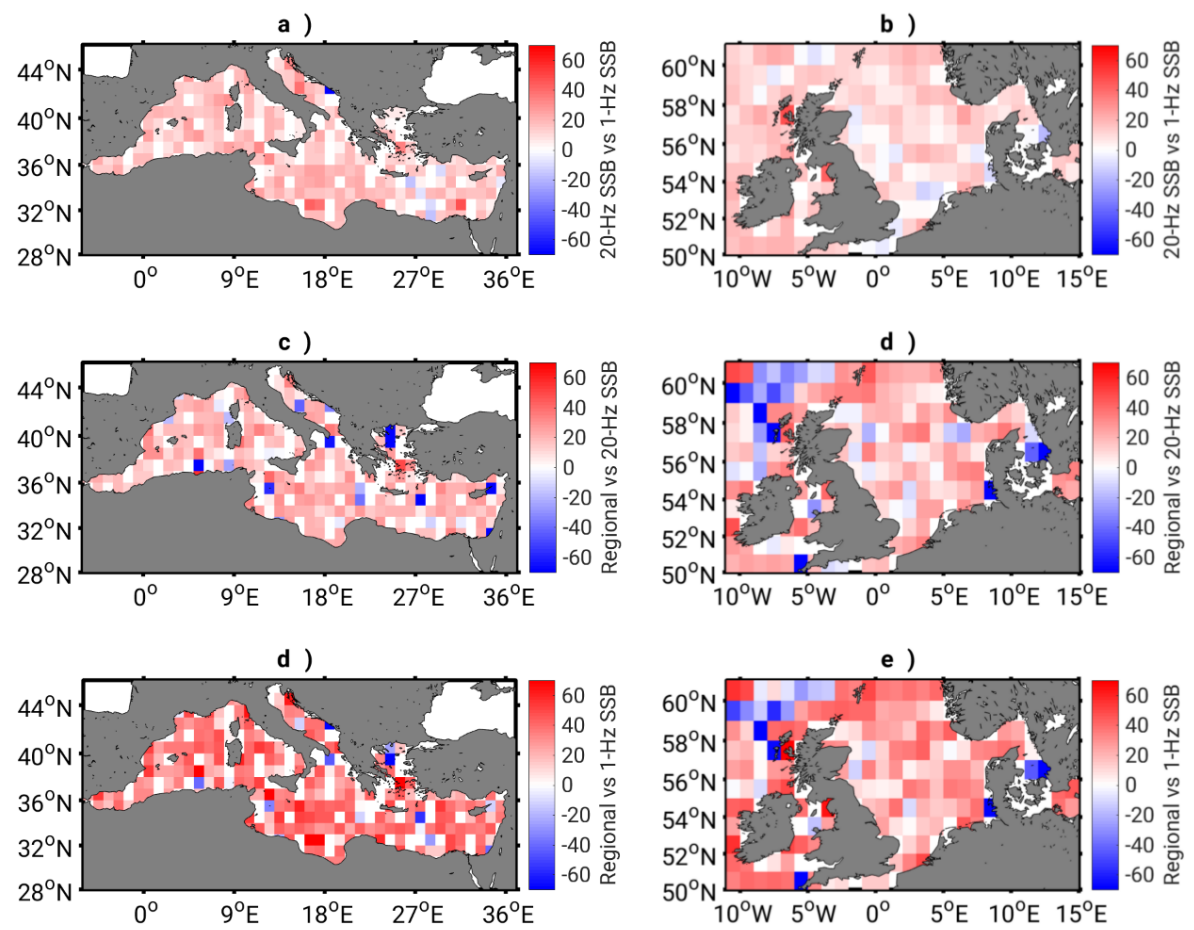

Figure 7: As in Figure 6, but the dataset used is ALES.

with the values showing a clear tendency to a larger magnitude at larger wave heights (see Figure 8). The application of $20-\mathrm{Hz}$ SSB corrections reduces the magnitude of this regression coefficient. A similar pattern is seen for the output of the ALES retracker: with a $1-\mathrm{Hz}$ SSB model applied, the median value of the scaling is -0.102 , but there is less variation with SWH in particular for SWH between 2 and $7 \mathrm{~m}$, due to the adaptive retracking window used by this retracker, whose width is tuned on the SWH value. Similar results are noted for the Mediterranean dataset, except that there were fewer observations for the domain $\mathrm{SWH}>8 \mathrm{~m}$.

The regression term $\beta$ represents a residual retracker-related noise, which is partly compensated for by the SSB correction. This analysis shows that applying SSB models at the full data rate and recomputing a regional model as described in this paper reduce the correlation between SLA and SWH estimation. 
a)

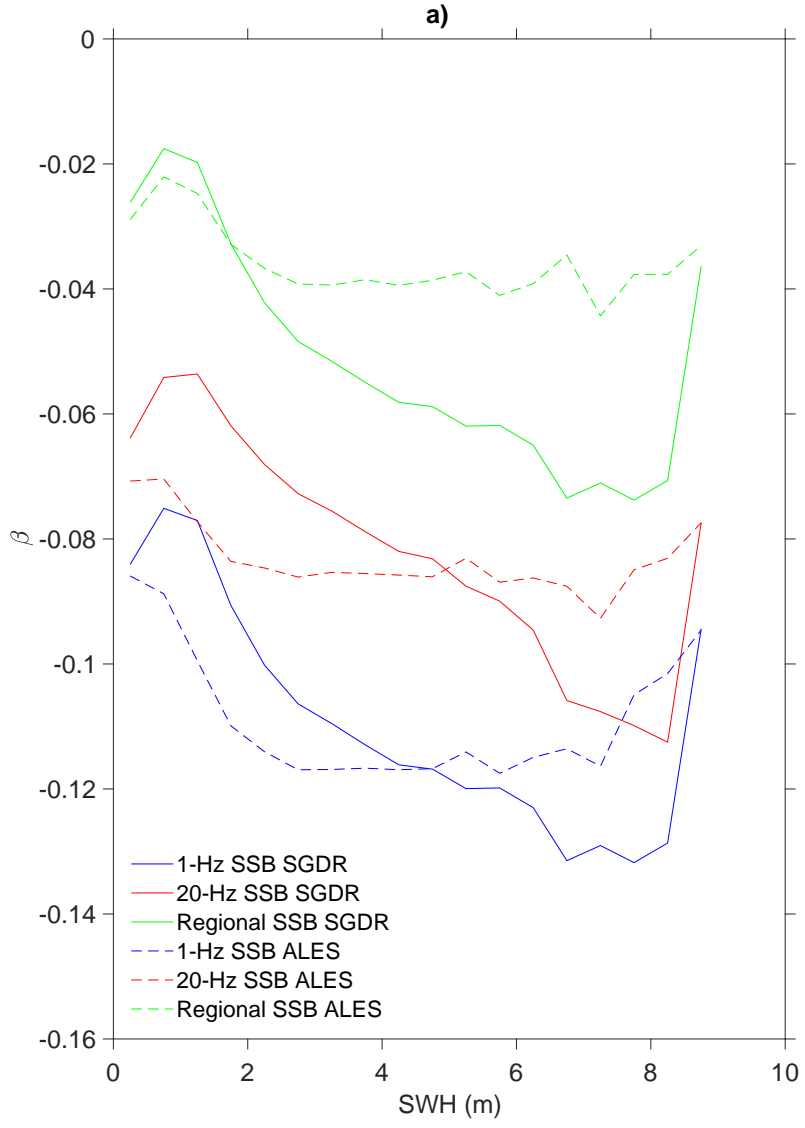

b)

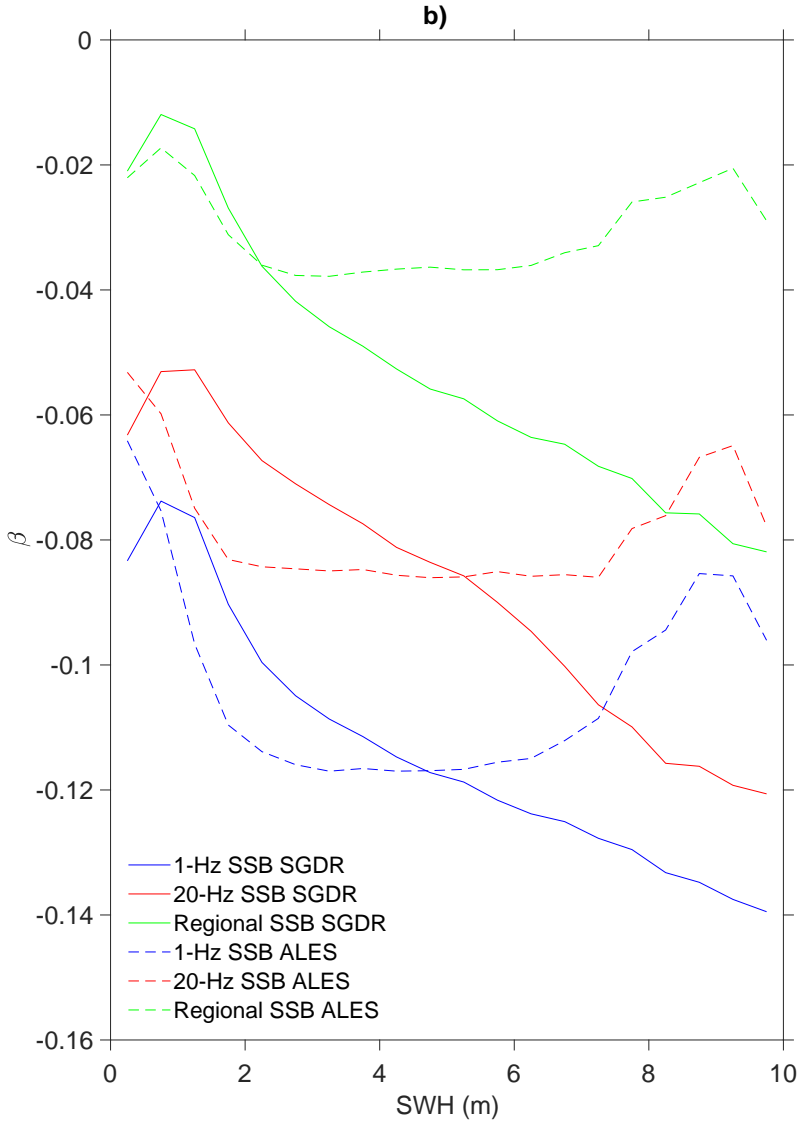

Figure 8: Variation of the regression coefficient, $\beta$ as a function of SWH using different SSB corrections analyzed in this study in Med (a) and NS (b). Continuous lines refer to SGDR data, while dashed lines refer to ALES data. The sea level anomalies were corrected with the original 1-Hz SSB correction (blue), with the 20-Hz SSB correction (red) and with the regional SSB correction (green). 


\section{Conclusions}

This study demonstrates, using Jason-1 mission as a testbed, that the combination of the use of HF estimations and a regional parametric approach provide a SSB correction that improves the precision of HF sea level data by more than one fourth with respect to the current standard.

We argued and justified that part of the reason lies in the suppression of most of the so-called "tracker bias", which is actually due to correlated errors in the retracking process and is therefore called "retracker-related noise" in this study following Zaron \& DeCarvalho (2016). This error is not correctly modeled in a LF SSB correction.

Another improvement is brought by a dedicated regional approach, which showed that the noise in sea level estimation, and consequently the recomputed SSB model, behaves differently in different regions, probably due to residual errors of different nature, which require further investigations.

One drawback of the methodology proposed here could be the following: if one assumes that the SSB estimation is related on one side to the real SWH and wind through a physical low-frequency relation and on the other side to the high-frequency errors in the estimation of SWH and wind, the empirical approach proposed in this work assumes that their combined effect can be modelled together. While this exploratory study demonstrates that this assumption produces more precise estimates than the current SSB model applied at $1-\mathrm{Hz}$, we cannot exclude that the separate treatment of the two components could generate an even better SSH estimation. The general aim of the research on SSB shall be therefore to work on a retracked dataset that is free from the retracker-related noise, in order to correct for the physical effects of the interaction between the radar signal and the waves. This is therefore one objective of our future work, which shall also further investigate regional differences, understand if the latter are present also when using a non-parametric approach and focus on high sea states, which are poorly represented in our model.

In conclusion, while providing a significantly more precise solution to exploit HF sea level data, this study gives robustness to previous theories on SSB, proposes a method to reduce the retracker-related noise alternative to Zaron \& DeCarvalho (2016) and provide an immediate improvement for the application of satellite altimetry in the North Sea and in the Mediterranean Sea. 


\section{Acknowledgements}

This work was partially funded by the Sea Level Climate Change Initiative, overseen by Jérôme Benveniste at the European Space Agency. The authors would like to thank Paolo Cipollini and Jesus Gómez-Enri for their suggestions and genuine interest.

\section{Bibliography}

Abdalla, S. (2012). Ku-band radar altimeter surface wind speed algorithm. Mar. Geod., 35, 276-298.

Amarouche, L., Thibaut, P., Zanife, O., Dumont, J.-P., Vincent, P., \& Steunou, N. (2004). Improving the Jason-1 ground retracking to better account for attitude effects. Mar. Geod., 27, 171-197.

Andersen, O., \& Scharroo, R. (2011). Range and geophysical corrections in coastal regions and implications for mean sea surface determination. In S. Vignudelli, A. Kostianoy, P. Cipollini, \& J. Benveniste (Eds.), Coastal Altimetry (pp. 103-146). Berlin Heidelberg: Springer-Verlag.

Birol, F., \& Delebecque, C. (2014). Using high sampling rate (10/20 hz) altimeter data for the observation of coastal surface currents: A case study over the northwestern Mediterranean Sea. J. Mar. Syst., 129, 318-333.

Birol, F., Fuller, N., Lyard, F., Cancet, M., Nino, F., Delebecque, C., Fleury, S., Toublanc, F., Melet, A., Saraceno, M. et al. (2017). Coastal applications from nadir altimetry: Example of the X-TRACK regional products. Adv. Space Res., 59, 936-953.

Carrère, L., \& Lyard, F. (2003). Modeling the barotropic response of the global ocean to atmospheric wind and pressure forcing-comparisons with observations. Geophys. Res. Lett., 30, 1275.

Chelton, D. B. (1994). The sea state bias in altimeter estimates of sea level from collinear analysis of TOPEX data. J. Geophys. Res. Oceans, 99, 24995-25008.

Cipollini, P., Benveniste, J., Birol, F., Fernandes, M., Obligis, E., Passaro, M., Strub, P., Valladeau, G., Vignudelli, S., \& Wlikin, J. (2017a). Satellite altimetry in coastal 
regions. In D. Stammer, \& A. Cazenave (Eds.), Satellite Altimetry over Oceans and Land Surfaces (pp. 343-380). New York: CRC Press.

Cipollini, P., Calafat, F. M., Jevrejeva, S., Melet, A., \& Prandi, P. (2017b). Monitoring sea level in the coastal zone with satellite altimetry and tide gauges. Surveys in Geophysics, (pp. 1-25). doi:10.1007/s10712-016-9392-0.

Elfouhaily, T., Thompson, D., Vandemark, D., \& Chapron, B. (1999). Weakly nonlinear theory and sea state bias estimations. J. Geophys. Res. Oceans, 104, 7641-7647.

Fernandes, M. J., Lázaro, C., Ablain, M., \& Pires, N. (2015). Improved wet path delays for all esa and reference altimetric missions. Remote Sensing of Environment, 169, $50-74$.

Fu, L., \& Cazenave, A. (Eds.) (2001). Satellite altimetry and earth sciences. A handbook of techniques and applications. volume 69. San Diego, CA: Academic.

Fu, L.-L., \& Glazman, R. (1991). The effect of the degree of wave development on the sea state bias in radar altimetry measurement. J. Geophys. Res. Oceans, 96, 829-834.

Garcia, E. S., Sandwell, D. T., \& Smith, W. H. (2014). Retracking Cryosat-2, Envisat and Jason-1 radar altimetry waveforms for improved gravity field recovery. Geophys. J. Int., (p. ggt469).

Gaspar, P., Labroue, S., Ogor, F., Lafitte, G., Marchal, L., \& Rafanel, M. (2002). Improving nonparametric estimates of the sea state bias in radar altimeter measurements of sea level. J. Atmos. Oceanic Tech., 19, 1690-1707.

Gaspar, P., Ogor, F., Le Traon, P.-Y., \& Zanife, O.-Z. (1994). Estimating the sea state bias of the TOPEX and POSEIDON altimeters from crossover differences. J. Geophys. Res. Oceans, 99, 24981-24994.

Gómez-Enri, J., Cipollini, P., Passaro, M., Vignudelli, S., Tejedor, B., \& Coca, J. (2016). Coastal altimetry products in the Strait of Gibraltar. IEEE Trans. Geosci. Remote Sens., 54, $5455-5466$. 
Labroue, S., Gaspar, P., Dorandeu, J., Zanife, O., Mertz, F., Vincent, P., \& Choquet, D. (2004). Nonparametric estimates of the sea state bias for the Jason-1 radar altimeter. Mar. Geod., 27, 453-481.

Legeais, J.-F., Ablain, M., Zawadzki, L., Zuo, H., Johannessen, J. A., Scharffenberg, M. G., Fenoglio-Marc, L., Fernandes, M. J., Andersen, O. B., Rudenko, S. et al. (2018). An improved and homogeneous altimeter sea level record from the esa climate change initiative. Earth System Science Data, 10, 281.

Pascual, A., Marcos, M., \& Gomis, D. (2008). Comparing the sea level response to pressure and wind forcing of two barotropic models: validation with tide gauge and altimetry data. J. Geophys. Res. Oceans, 113.

Passaro, M. (2017). COSTA v1.0: DGFI-TUM along track sea level product for ERS2 and Envisat (1996-2010) in the Mediterranean Sea and in the North Sea, links to data sets in NetCDF format. Deutsches Geodaetisches Forschungsinstitut der Technischen Universitaet Muenchen, PANGAEA, . doi:https://doi .org/10.1594/PANGAEA . 871920.

Passaro, M., Cipollini, P., Vignudelli, S., Quartly, G., \& Snaith, H. (2014). ALES: A multi-mission subwaveform retracker for coastal and open ocean altimetry. Remote Sens. Environ., 145, 173-189.

Passaro, M., Dinardo, S., Quartly, G. D., Snaith, H. M., Benveniste, J., Cipollini, P., \& Lucas, B. (2016). Cross-calibrating ALES Envisat and Cryosat-2 Delay-Doppler: A coastal altimetry study in the Indonesian Seas. Adv. Space Res., 58, 289303.

Passaro, M., Rose, S., Andersen, O., Boergens, E., Calafat, F., Dettmerring D., \& Benvensite, J. (2018). ALES+: Adapting a homogenous ocean retracker for satellite altimetry to sea ice leads, coastal and inland waters. Remote Sens. Environ., 211, 456-471. doi:10.1016/j.rse.2018.02.074.

Picot, N., Case, K., Desai, S., \& Vincent, P. (2003). AVISO and PODAAC user handbook. IGDR and GDR Jason products. SMM_MU_M5_OP_13184_CN (AVISO) JPL D_21352 $(P O D A A C)$. 
Pires, N., Fernandes, M. J., Gommenginger, C., \& Scharroo, R. (2016). A conceptually simple modeling approach for Jason-1 sea state bias correction based on 3 parameters exclusively derived from altimetric information. Remote Sens., 8, 576.

Quartly, G., Legeais, J.-F., Ablain, M., Zawadzki, L., Fernandes, M., Rudenko, S., Carrère, L., García, P. N., Cipollini, P., Andersen, O., Poisson, J.-C., Mbajon Njiche, S., Cazenave, A., \& Benveniste, J. (2017). A new phase in the production of qualitycontrolled sea level data. Earth System Science Data, 9, 557-572.

Quartly, G., Smith, W., \& Passaro, M. (2018). Removing intra-1 hz covariant error to improve altimetric profiles of $\sigma^{0}$ and sea surface height (submitted), IEEE Trans. Geosci. Remote Sens..

Quartly, G. D. (2009). Optimizing $\sigma^{0}$ information from the Jason-2 altimeter. IEEE Geosci. Remote Sens. Lett., 6, 398-402.

Quartly, G. D. (2010). Hyperbolic retracker: Removing bright target artefacts from altimetric waveform data. In ESA SP-686, Living Planet Symposium 2010, Bergen, Norway, (28 June - 2 July 2007) ESA Publication, SP-686. Noordwijkerhout, NL: ESA.

Quartly, G., Smith, W., \& Passaro, M. (2016). Intra-1 hz Correlations, presented at the Ocean Surface Topography Science Team Meeting, La Rochelle, France, 1-4 November 2016. Available from https://mediatum.ub.tum.de/doc/1338249/1338249.pdf.

Sandwell, D. T., \& Smith, W. H. (2005). Retracking ERS-1 altimeter waveforms for optimal gravity field recovery. Geophys. J. Int., 163, 79-89.

Thibaut, P., Poisson, J., Bronner, E., \& Picot, N. (2010). Relative performance of the MLE3 and MLE4 retracking algorithms on Jason-2 altimeter waveforms. Mar. Geod., 33, 317-335.

Tran, N., Labroue, S., Philipps, S., Bronner, E., \& Picot, N. (2010). Overview and update of the sea state bias corrections for the Jason-2, Jason-1 and TOPEX missions. Mar. Geod., 33, 348-362. 
482

Tran, N., Vandemark, D., Labroue, S., Feng, H., Chapron, B., Tolman, H.L., Lambin, J., \& Picot, N. (2010). Sea state bias in altimeter sea level estimates determined by combining wave model and satellite data . J.Geophys.Res., 115, C03020.

Vandemark, D., Tran, N., Beckley, B., Chapron, B., \& Gaspar, P. (2002). Direct estimation of sea state impacts on radar altimeter sea level measurements. Geophys. Res. Lett., 29.

Zaron, E. D., \& DeCarvalho, R. (2016). Identification and reduction of retracker-related noise in altimeter-derived sea surface height measurements. J. Atmospheric Ocean. Technol., 33, 201-210. 